\title{
Factors Associated with Psychiatric Ward Admission in the Emergency Department after a Suicide Attempt: the Risk-Rescue Rating Scale (RRRS) and Biochemical Markers
}

\author{
Minho Cho ${ }^{1}$, Hyejin Kim ${ }^{1, *}$
}

\author{
${ }^{1}$ Department of Emergency Medicine, \\ Inje University Sanggye Paik Hospital, \\ Seoul, Korea \\ *Correspondence \\ veauvoir@paik.ac.kr \\ (Hyejin Kim)
}

\begin{abstract}
Objective: This study was conducted to understand factors associated with psychiatric ward admission for patients referred to the emergency department after a suicide attempt. Methods: Medical records of patients referred to the emergency department (ED) after attempting suicide were retrospectively reviewed. Two groups were formed: the discharged (DC) group and the psychiatric ward (PW) admission group. All statistical analyses, including univariate and logistic regression analyses, were performed using SPSS 18.0 software, with $\mathrm{p}<0.05$ considered as statistically significant. Results: This study included 486 patients, $92.0 \%(n=447)$ in the DC group and $8.0 \%(n=39)$ in the PW admission group. Multivariate logistic regression analysis showed that predictors of PW admission included in referral during the daytime $(\mathrm{OR}=$ $3.39, \mathrm{p}<0.01)$, history of depression $(\mathrm{OR}=3.13, \mathrm{p}=0.01)$, the Risk-Rescue Rating Scale (RRRS) $(\mathrm{OR}=1.14, \mathrm{p}<0.01)$, drinking status $(\mathrm{OR}=0.22, \mathrm{p}<$ 0.01). Conclusions: We demonstrated that RRRS could be used to determine PW admission for patients referred to ED after suicide attempts. Furthermore, history of daytime visiting, non-drinking status, and history of depression were identified as factors relating to PW admission. Active and careful attention is recommended for patients with factors relating to PW admission among those who were voluntarily discharged.
\end{abstract}

\section{Keywords}

Suicide, Emergency service, Hospital, Biomarkers

\section{Introduction}

Suicide is a serious issue at the individual and societal levels, and is a major cause of death among adolescents and young adults worldwide. Mortality from suicide has been increasing since the 2008 financial crisis. A recent World Health Organization (WHO) report highlighted the need to improve the public health perception of suicide by prioritizing suicide prevention in public health agendas owing to an increase in suicide among middle-aged people worldwide [1].

In Korea, the emergency department (ED) is the initial stop following a suicide attempt due to the need for immediate medical treatment, but it can also offer primary medical care or an outpatient clinic when no alternative services are available [2]. Therefore, the ED provides primary care to patients who have attempted suicide and determines whether these patients will be hospitalized or placed under short-term follow-up care as outpatients [3].

Among patients who visited the hospital after a suicide attempt, 80\% received emergency hospitalization. In addition, all patients admitted for suicide attempts in France receive psychiatric assessments [4]. These assessments provide an opportunity to evaluate the suicide attempt, initiate treatment, and prevent future self-harm. Furthermore, when an assessment of suicidal behavior is made during a visit to the ED, it can play an important role in preventing subsequent suicide attempts [5]. 
TA B LE 1. Risk rating.

\begin{tabular}{|c|c|c|c|}
\hline \multirow[t]{2}{*}{ Risk factors } & \multicolumn{3}{|c|}{ Points } \\
\hline & 1 & 2 & 3 \\
\hline Agent used & Ingestion, cutting, stabbing & $\begin{array}{l}\text { Drowning, Asphyxia, } \\
\text { strangulation }\end{array}$ & Jumping, shooting \\
\hline Impaired consciousness & Non in evidence & Confusion, semi-coma & Coma, deep coma \\
\hline Lesions/toxicity & Mild & Moderate & Severe \\
\hline Reversibility & $\begin{array}{l}\text { Good, complete recovery } \\
\text { expected }\end{array}$ & $\begin{array}{c}\text { Fair, recovery expected with } \\
\text { time }\end{array}$ & $\begin{array}{l}\text { Poor, residuals expected if } \\
\text { recovery occurs }\end{array}$ \\
\hline Treatment required & $\begin{array}{c}\text { First aid, emergency room } \\
\text { care }\end{array}$ & $\begin{array}{c}\text { Admission, routine } \\
\text { treatment }\end{array}$ & $\begin{array}{c}\text { Intensive care, special } \\
\text { treatment }\end{array}$ \\
\hline
\end{tabular}

TA B L E 2. Rescue rating.

\begin{tabular}{|c|c|c|c|}
\hline \multirow[t]{2}{*}{ Rescue factors } & \multicolumn{3}{|c|}{ Points } \\
\hline & 3 & 2 & 1 \\
\hline Location & Familiar & Non-familiar, non-remote & Remote \\
\hline $\begin{array}{l}\text { Person initiating rescue (In case of self-rescue, } \\
\text { the rescue score }=5 \text { ) }\end{array}$ & Key person & Professional & Passer by \\
\hline Probability of discovery by any rescuer & High, almost certain & Uncertain discovery & Accidental discovery \\
\hline Accessibility to rescue & Asks for help & Drops clues & Does not ask for help \\
\hline Delay until discovery & Immediate 1 hour & Less than 4 hours & Greater than 4 hour \\
\hline \multirow[t]{2}{*}{ Undue delay between discovery and treatment } & $\mathrm{YES}=-1$ & $\mathrm{YES}=-1$ & $\mathrm{YES}=-1$ \\
\hline & $\mathrm{No}=0$ & $\mathrm{No}=0$ & $\mathrm{No}=0$ \\
\hline
\end{tabular}

The decision to hospitalize or discharge patients who have attempted suicide is based on such evaluations. While there are many guidelines on performing suicide risk assessments, none are universally accepted [6-8]. The American Psychiatric Association (APA) guidelines for deciding hospitalization after a suicide attempt are based on intention and severity, two factors that influence one another [9]. The most common scales for assessing suicide severity are the Risk-Rescue Rating Scale (RRRS) and the Self-inflicted Injury Severity Form (SISF) [10], while suicidal intent is primarily evaluated using the Suicide Intent Scale (SIS) [2]. These scales have been used to determine the risk of subsequent suicide attempts and to measure the overall suicide mortality rate [3]. However, there is insufficient research on the usefulness of these scales in determining hospitalization during an ED visit.

Hospitalization following a suicide attempt is generally advised when there is an imminent risk of suicide. Policies relating to psychiatric ward admission for treatment of suicidal behaviors are associated with clinical factors such as older age, sex (male), poor health, mental illness, high suicidal intent, suicidal ideation, previous psychiatric hospitalization, surviving a suicide attempt, and low expectations of patient discovery after a suicide attempt. Additionally, these factors include violent or pre-planned suicide attempts, precautions to avoid rescue, increased mental suffering, regret over survival, limited social support, impulsive actions, and clear rejection of help. Hospitalization in departments other than the psychiatric ward is recommended when there is a change in level of consciousness due to metabolic, toxic, infectious, or other causes [4].

Previous findings on the biological characteristics of suicide attempt history suggest that $\mathrm{C}$-reactive protein (CRP) value could be a characteristic factor related to vulnerability to suicidal behavior. Furthermore, it has been determined that inflammatory factors in individuals who demonstrate suicidal behaviors could lead to subsequent suicide attempts. Understanding these factors, therefore, presents new opportunities for developing treatment for patients who exhibit suicidal behaviors [5].

Inflammation is known to play a potentially critical role in suicidal behaviors [6]. CRP, Neutrophil-to-Lymphocyte Ratio (NLR), Platelet-to-Lymphocyte Ratio (PLR), and Systemic Immune Inflammation Index (SIII) are commonly used as biological markers of inflammation and can be obtained from hemogram parameters, which are used to predict and diagnose many diseases [7]. However, hemogram results are rarely used to determine the vulnerability of psychiatric patients to suicidal behaviors or hospitalization following a suicide attempt [8].

Regardless of whether multiple methods have been used to determine severity of a suicide attempt in deciding hospitalization or discharge, patients may be discharged against the advice, recommendations, or treatment plans of medical staff. In particular, recent discharges of a growing proportion of psychiatric patients make it difficult to accurately 
TA B L E 3. Patient characteristics stratified by discharge (DC), psychiatric ward (PW) admission after suicide attempts.

\begin{tabular}{|c|c|c|c|}
\hline Factors & DC group $(n=447)$ & PW group $(n=39)$ & $\chi^{2}$ or t-test \\
\hline Sex (male) & $143(32.0)$ & $14(35.9)$ & 0.25 \\
\hline Age (years) & $37.2 \pm 18.5$ & $33.8 \pm 16.3$ & 1.235 \\
\hline Visited during the daytime & $72(16.1)$ & $17(43.6)$ & $18.111 * * *$ \\
\hline Mental status (altered mental status) & $109(24.5)$ & $8(20.5)$ & 0.348 \\
\hline Educational level (middle school or less) & $93(27.4)$ & $11(28.9)$ & 0.39 \\
\hline Marital status (married) ${ }^{\dagger}$ & $150(37.9)$ & $10(26.3)$ & 1.992 \\
\hline Religion & $77(27.2)$ & $11(32.4)$ & 0.401 \\
\hline Occupation (employed) & $229(61.1)$ & $21(56.8)$ & 0.262 \\
\hline Income ( $<2.5$ million KRW per month) & $173(52.7)$ & $15(42.9)$ & 1.312 \\
\hline Drinking & $188(43.4)$ & $4(10.5)$ & $15.652 * * *$ \\
\hline Attempted suicide before & $186(45.0)$ & $16(41.0)$ & 0.232 \\
\hline Met a psychiatrist before & $200(58.0)$ & $23(69.7)$ & 1.712 \\
\hline Admitted to a psychiatric ward before & $74(18.9)$ & $11(28.2)$ & 1.949 \\
\hline Taking psychiatric medicine & $116(40.0)$ & $8(38.1)$ & 0.043 \\
\hline Family history of psychiatric disease & $95(24.5)$ & $11(28.2)$ & 0.254 \\
\hline $\begin{array}{l}\text { Physical status Healthiness Acute disease } \\
\text { Chronic disease }\end{array}$ & $331(79.6) 2(0.5) 83(20.0)$ & $33(86.8) 1(2.6) 4(10.5)$ & 4.429 \\
\hline $\begin{array}{l}\text { Presumptive diagnosis MDD Psychiatric } 28 \\
\text { disorder other than MDD Uninterrupted or } \\
\text { impossible to diagnose }\end{array}$ & 86 (64.0) 48 (10.7) $113(25.3)$ & $27(69.2) 12(30.8) 0(0)$ & $24.478 * * *$ \\
\hline Plan about suicide attempt & $36(8.9)$ & $6(15.8)$ & 1.911 \\
\hline Suicidal ideation when visiting ER & $182(46.3)$ & $21(58.3)$ & 3.345 \\
\hline Risk rescue rating & $33.4 \pm 6.1$ & $38.3 \pm 6.5$ & $-4.307 * * *$ \\
\hline White Blood Cells $(/ \mu \ell)$ & $7.904 .5 \pm 2.678 .3$ & $9.154 .2 \pm 5.022 .8$ & -1.509 \\
\hline Hemoglobin $(g / d \ell)$ & $13.4 \pm 1.7$ & $13.2 \pm 1.7$ & 0.779 \\
\hline $\mathrm{CRP}(\mathrm{mg} / \mathrm{d} \ell)$ & $0.37 \pm 1.19$ & $0.36 \pm 0.79$ & 0.059 \\
\hline SBP (mmHg) & $125.6 \pm 53.8$ & $122.4 \pm 26.3$ & 0.646 \\
\hline DBP (mmHg) & $75.9 \pm 24.6$ & $75.4 \pm 13.5$ & 0.187 \\
\hline HR (beats/min) & $91.0 \pm 21.5$ & $89.0 \pm 25.3$ & 0.474 \\
\hline NLR & $3.1 \pm 2.7$ & $3.8 \pm 3.9$ & -1.143 \\
\hline Platelet $(\times 1.000)$ & $258.5 \pm 78.5$ & $263.8 \pm 72.1$ & -0.43 \\
\hline SIII $(\times 1000)$ & $776.7 \pm 635.7$ & $997.2 \pm 957.0$ & -1.386 \\
\hline PLR & $145.7 \pm 110.7$ & $154.5 \pm 57.4$ & -0.796 \\
\hline
\end{tabular}

Data are presented as frequency (percentage) or mean \pm standard deviation.

†The variable "marital status" had three values: married, registered relationship, and de facto relationship. ***; p < 0.001. MDD (major depressive disorder), ER (emergency room), Systolic blood pressure (SBP), Diastolic blood pressure $(D B P)$, Heart rate (HR), C-reactive protein (CRP), Neutrophil-to-lymphocyte ratio (NLR), Platelet-to-lymphocyte ratio (PLR), Systemic immune inflammation index (SIII).

assess and plan for future treatment of ED patients admitted for suicide attempts.

Voluntary discharge is a natural result of greater treatment choices as well as increased patient autonomy in determining treatment. However, this can cause dissatisfaction in medical staff engaged in treating patients, and may lead to harmful consequences resulting from incomplete treatment [9]. The most significant issue with voluntary discharge is that the rate of returning to the hospital with the same diagnosis within 15 days of discharge is seven times higher than that of patients receiving standard treatment $[10,11]$. Particularly, the voluntary discharge of a patient 
TA B L E 4. Multiple regression analysis for predicting admission to the psychiatric ward of patients at the emergency department after suicide attempts.

\begin{tabular}{lccc} 
& OR & $\mathbf{9 5 \%}$ CI & p value \\
\hline MDD (reference value: uninterrupted or impossible to diagnose) & 3.13 & $1.30-7.49$ & 0.011 \\
Referral during the daytime & 3.39 & $1.48-7.75$ & 0.004 \\
Risk rescue rating score & 1.14 & $1.07-1.21$ & $<0.001$ \\
Drinking & 0.22 & $0.07-0.66$ & 0.007 \\
\hline
\end{tabular}

Major depressive disorder (MDD), CI (confidence interval).

who attempts suicide is a serious problem as it may have irreversible consequences. Voluntary discharge by patients requiring psychiatric diagnosis or treatment is known to be much higher than that of internal medicine or surgery patients. General wards of university hospitals have shown voluntary discharge rates of $0.7 \%$, while rates in pediatric psychiatric outpatient clinics have been as high as 51\% [12]. Furthermore, voluntary discharge was between 3 - 51\% higher in psychiatric patients (averaging 17\%) compared to internal medicine patients [9].

Therefore, this study was conducted to understand factors related to psychiatric ward admission for patients requiring psychiatric care and treatment, excluding those in other departments. Understanding these factors could help establish standards of safe discharge of patients and possibly minimize the voluntary discharge of those who attempted suicide. In addition, we note that few studies have used RRRS and biomarkers to investigate psychiatric ward admission of patients who have attempted suicide.

\section{Methods}

\subsection{Study design and setting}

This retrospective study involves patients who visited the ED of the general hospital in a large urban city in South Korea from March 2017 to December 2019 following a suicide attempt. Our primary dependent variable was the disposition of patients who attempted suicide after being referred to the ED. These patients were either discharged to their homes (DC group) or admitted to the psychiatric ward (PW group). This study was approved by the Institutional Review Board (IRB), conforming to the recommendations of the Declaration of Helsinki.

\subsection{Selection of Participants}

An average of 65,000 patients visit the hospital in South Korea every year, including more than 70 who are admitted to the psychiatric ward. During the study period, 642 patients were referred to the ED following a suicide attempt. The final study sample included 486 patients after the exclusion of 156. The excluded patients included: nine who had their registrations canceled because their request for care was refused (patients signed voluntary discharge forms), eight who left the hospital against medical advice after registration, 21 who were referred to psychiatric hospitals after ED evaluation, and 27 death cases (i.e. 18 on arrival, two at the ED, and seven during hospitalization). The other excluded patients were 62 admitted to the intensive care unit for medical or surgical treatment and 29 admitted to the general ward, instead of the psychiatric ward.

Patients were also excluded if 1) they were accidentally poisoned with no suicidal intention as determined by an emergency medicine physician; and 2) they were referred to an outpatient psychiatry clinic other than the ED after a suicide attempt (Fig. 1).

As shown in Fig. 1, participants were then classified into two groups: discharged patients (DC group), and those admitted to the psychiatric ward (PW group).

\subsection{Instruments}

All patients who visited the ED after a suicide attempt were referred to the concerned case management team. The initial assessment forms were designed by this team under the supervision of a psychiatrist at the hospital. Patients who agreed to be managed by the case management team responded to all items on their forms. Meanwhile, the forms for those who did not agree were incomplete, and we had to obtain as much information as possible from their electronic medical records (EMR).

The initial assessment forms included information about participants' marital status, religion, employment status, income level (with reference to the average monthly income of Korean workers, classified into $\geq 2.5$ million KRW and $<2.5$ million KRW), education level (with reference to the mandatory education in Korea, classified into middle school graduate or lower and high school graduate or higher), suicide attempt method, drinking status, history of prior suicide attempts, history of psychiatric care, psychiatric drug use, history of admission to psychiatric ward, family psychiatric history, suicide attempt plan, and suicidal ideation during treatment.

The following data was collected from the patients' EMR: sex; age; vital signs (systolic blood pressure, diastolic blood pressure, and heart rate); level of consciousness (alert: alert mentality; drowsy, stupor, and coma: altered mentality); type of referral (during business hours, i.e. 9AM to 5PM on weekdays, or during off hours $)$; RRRS scale $=[$ Risk rating $/($ Risk rating + Rescue rating) $\times 100$ (risk and rescue ratings are presented in Table 1 and Table 2); ED outcomes (PD admission, and discharge); and physical status at the time of referral to 


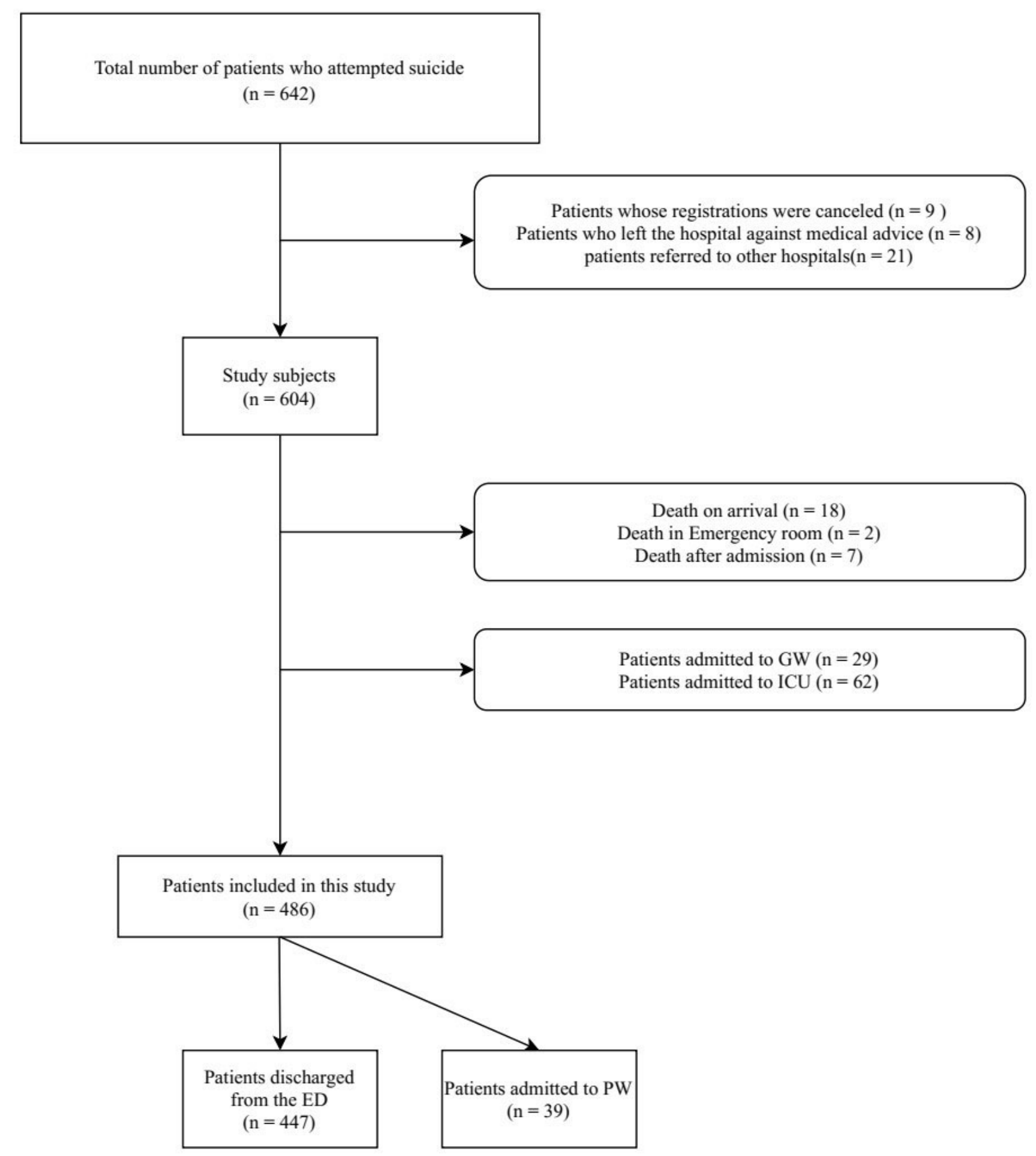

Emergency department(ED)

Psychiatric ward (PW)

General ward(GW)

Intensive care unit(ICU)

\section{F I G U R E 1. Patient selection flow chart.}

the ED (chronic disease, acute disease, and physically healthy). In addition, white blood cell (WBC) count, Hemoglobin, Platelet, and CRP were measured through blood sampling with venipuncture. Hemogram parameters were used to compute NLR, PLR, and SIII (NLR =
Neutrophils/Lymphocytes; PLR = Platelets/Lymphocytes; and SIII $=$ Platelets $\times$ Neutrophils/Lymphocytes). The presumptive diagnosis for each patient was confirmed only after an interview with a psychiatrist for patients who had requested a psychiatric consultation. The 
presumptive diagnosis for patients who had attempted suicide and refused psychiatric consultation was classified as uninterrupted. Past psychiatric diagnoses of the uninterrupted patients were used based on a statement from the patient or their guardian. For statistical convenience, presumptive psychiatric diagnosis was divided into three categories: depression, psychiatric disease other than depression, and no intervention or impossible to diagnose. Data were collected and RRRS determined by a senior emergency medicine resident under the supervision of an emergency medicine specialist.

\subsection{Statistical analysis}

We performed univariate analysis of the differences in demographic variables, a clinical scale (i.e. RRRS), and biomarkers (i.e. WBC, CRP, NLR, PLR, and SIII) among the two groups of patients. Nominal and continuous variables were analyzed with Chi-square tests or Fisher's exact test and the independent t-test or Mann-Whitney U-test, respectively. Variables of significant differences in univariate analysis were included in multiple logistic regression models.

Comparisons were made between the DC group (reference) and the PW group (target). A ROC curve analysis was performed to identify possible predictors of PW admission with the DC group as reference. In addition, the optimal area under the ROC curve (AUC), sensitivity, and specificity were determined based on the cut-off values for the relevant parameters. Significance was set at $p<0.05$, and all statistical analyses were performed using the SPSS 18.0 (SPSS Inc., Chicago, IL, USA) software.

\section{Results}

\subsection{Baseline Characteristics and Comparison of the DC and PW groups}

Baseline characteristics of the 486 study participants are presented in Table 3. The mean age of the enrolled patients was 36.9 years, and $32.3 \%$ were men. Only 89 (18.3\%) patients visited the emergency room during the daytime and 397 (81.7\%) patients visited during off hours. The variable "marital status" comprised the values married, registered relationship, and de facto relationship; 160 (36.9\%) patients were classified as married and $326(63.1 \%)$ were classified as single. The presumptive diagnosis was classified into three categories: major depressive disorder (MDD; $\mathrm{n}=$ $313,64.4 \%$ ), psychiatric disorders other than MDD ( $\mathrm{n}=$ $60,12.3 \%$ ), and uninterrupted or impossible to diagnose (n $=113,23.3 \%$ )

Of the total 486 patients who attempted suicide, 447 $(92.0 \%)$ were discharged and only $39(8.0 \%)$ were admitted to the psychiatric ward. Significant differences were shown between the two groups in presumptive diagnosis, drinking status, daytime visit, and RRRS (Table 3).

\subsection{Multiple regression analysis for predicting admission to the psychiatric ward after a suicide attempt}

We conducted multiple regression analysis to predict PW admission for patients admitted to the ED following a suicide attempt. The factors that showed significant differences between the PW and DC groups were history of depression (reference value: uninterrupted or impossible to diagnose $)(\mathrm{OR}=3.13,95 \% \mathrm{CI}: 1.3-7.49$; $\mathrm{p}<0.01)$; daytime visit $(\mathrm{OR}=3.39,95 \% \mathrm{CI}: 1.48-7.75 ; \mathrm{p}<0.01)$; drinking status $(\mathrm{OR}=0.22,95 \% \mathrm{CI}: 0.07-0.66 ; \mathrm{p}<0.01)$; and RRRS scores (OR $=1.14,95 \% \mathrm{CI}$ : $1.07-1.21$; p < 0.01) (Table 4).

\section{Discussion}

Voluntary discharge from medical treatment is a widely used concept, but the practical criteria for defining voluntary discharge varies across studies. These variations include patients who sign their voluntary discharge form, those who return home earlier than scheduled in the treatment plan, and discharges against the medical advice of attending physicians.

About $76 \%$ of patients (339) returned to the ED after a suicide attempt and were discharged after signing a voluntary discharge form; 182 signed a voluntary discharge form after being admitted to the PW; 265 rejected psychiatric consultation altogether, and among them, only 157 signed a voluntary discharge form. All patients who had psychiatric consultations signed voluntary discharge forms before returning home. However, patients who rejected the psychiatric consultation only signed voluntary discharge forms when they attempted suicide using life-threatening methods, had a previous history of suicide attempts, and exhibited an unstable psychological condition during admission to the ED under the subjective judgment of the ED physician. This indicates that the criteria for voluntary discharge includes rejection of the psychiatric consultation recommended by the ED physician, even if the patient does not sign the voluntary discharge form. In fact, patients admitted to the ED after a suicide attempt cannot be seen as fully evaluated or having an improved condition during their stay in the ED. Ultimately, those patients who attempt suicide and return home after ED treatment can be considered voluntarily discharged in a broader sense.

This study excluded ED patients who had attempted suicide and were admitted to the ICU for medical treatment of physical injuries. Also excluded were those patients who had attempted suicide but were placed in the internal medicine or surgery departments, and the patients whose biochemical markers were influenced by physical injuries sustained during the suicide attempt.

Characteristics of patients admitted to the PW after a suicide attempt were compared with those of the DC group using a clinical scale and biological markers. Based on these results, it was anticipated that medical staff could provide more attentive and proactive psychiatric assessments 
and recommendations for hospitalization for the patients who were voluntarily discharged and showed similar characteristics as the patients who were admitted to the PW. This will provide opportunities for psychiatric assessment and treatment of those patients who attempt suicide, and could ultimately prevent subsequent suicide attempts.

Studies have suggested that clinical scales can determine patient disposition [3]. They have confirmed that clinical scales implemented during emergency situations can help predict hospitalization of patients who have attempted suicide. Among the scales used in the study, RRRS (risk-rescue rating scale) showed superior predictability. Furthermore, combining scales was more important than implementing each scale alone in showing better predictive performance [13].

Our study examined only RRRS along different clinical scales and confirmed that it was an important factor in determining PW admission for those who attempted suicide. When RRRS was applied alone and the RRRS value of 35.5 was set as the cut off, a sensitivity of $62.9 \%$ and a specificity of $66.3 \%$ (AUC $=0.706, \mathrm{p}<0.001)$ were shown.

A previous study regarding the use of NLR, PLR, and SIII proportions to identify depression patients who were expected to attempt suicide indicated that NLR and other biochemical markers from hemograms were higher in patients with depression compared to patients who attempted suicide, but these results were not statistically significant. The study concluded that biochemical markers, such as NLR, PLR, and SIII, cannot be used in predicting suicide attempts [8].

In the present study, no biochemical markers showed statistically significant differences between patients who were voluntarily discharged and patients admitted into the PW.

Compared to the discharge group, the proportion of patients visiting between the normal working hours of 9 AM to $5 \mathrm{PM}$ was notably higher in the PW group $(\mathrm{OR}=3.39, \mathrm{p}<$ $0.01)$. This could be because patients visiting during normal working hours have a better objective perspective about their situation and a higher willingness for treatment. In the case of patients admitted for a suicide attempt outside of normal working hours (i.e. evening, night, early morning, and weekend), their suicide attempts could have been led by mood changes attributed to time of day. Another possibility is that these patients considered their situation to be a simple deviation from daily routine or not serious, which could had led to a lack of motivation for self-treatment.

The number of patients admitted who were not under the influence of alcohol was higher in the PW group compared to the DC group, and these results were statistically significant $(\mathrm{OR}=4.7, \mathrm{p}<0.01)$, similar to the findings of another study [14]. This previous study showed that patients with alcohol-related suicide attempts had voluntary discharge rates twice as high as patients without alcoholrelated suicide attempts.

The present results also corroborate another study which found that a high proportion of patients being voluntarily discharged were using alcohol or other drugs, and emphasized that it was important to obtain their voluntary discharge forms when they were sent home [10]. In these cases, it is likely that the suicide attempt occurred under the influence of alcohol if the patient or their guardian considered the attempt to be simply impulsive, or if they made a poor decision regarding the need for psychiatric treatment following the suicide attempt [15].

The number of patients with a history of depression was statistically significantly higher in the PW group compared to the DC group, and these results are consistent with previous studies [16]. A previous history of depression indicated a high rate of recurrent suicide attempts, but also indicated a high rate of acceptance of the psychiatric interview and education [17]. This may be due to physician concerns over recording a psychiatric diagnosis for patients with no previous history of depression, and for patients already diagnosed with depression. It is possible that patients were less resistant to psychiatric care and exhibited a high level of willingness to receive treatment. Considering studies that show hospital practices influence psychiatric consultations of patients that attempt suicide [18], it is possible that the consultation practices of the research institution participating in this study may have influenced the results. Furthermore, it is possible that the medical staff considered patients with a history of depression to be a high-risk group, and more actively offered diagnosis and hospitalization for such patients.

The limitations of this study are as follows. First, the study was conducted at a single center. The institution where this research took place is located in a residential area in the vicinity of a large city, and multiple similarly sized institutions exist nearby. Therefore, we cannot rule out the possibility that patients who attempted suicide may have been dispersed and admitted to nearby hospitals.

Second, the institution where this research took place provides consultation and support services for all patients admitted for a suicide attempt. Patients who agreed to receive these services had completed the initial assessment items. However, some patients who did not agree to receive these services declined to respond to the initial assessment. Although the missing data were supplemented with EMR as much as possible, the fact that these assessments were not completed could also constitute a study limitation.

Third, a high number of participants rejected psychiatric consultation. Therefore, we were unable to properly conduct the evaluation of patient insights about suicide attempts.

Fourth, blood tests were not completed for all patients. Blood tests were not conducted when patients had attempted suicide through stabbing or simple lacerations. In addition, blood tests were not carried out when the patients strongly refused them.

Fifth, the level of inflammation may have been affected by numerous factors, such as standard medications, as well as the impact of diseases (i.e. cancer, infections) on patient health. Therefore, the baseline values for the patient may 
differ. We suggest that a similar study should be conducted in the future within a larger, multicentered, prospective design.

We demonstrated that the RRRS could be used to determine PW admission for patients referred to the ED after a suicide attempt. Furthermore, a history of visiting the ED during the daytime, non-drinking status, and a history of depression were identified as factors related to PW admission. It is important to note that active and careful attention is required for patients with factors related to $\mathrm{PW}$ admission among those who were voluntarily discharged.

\section{ACKNOWLEDGMENTS}

This work was supported by the 2018 Inje University research grant.

\section{CONFLICT OF INTEREST}

The authors declare no conflict of interest.

\section{REFERENCES}

[1] Baca-García E, Diaz-Sastre C, Resa EG, Blasco H, Conesa DB, SaizRuiz J, et al. Variables associated with hospitalization decisions by emergency psychiatrists after a patient's suicide attempt. Psychiat Serv. 2004;55:792-7.

[2] Hasley JP, Ghosh B, Huggins J, Bell MR, Adler LE, Shroyer ALW. A review of "suicidal intent" within the existing suicide literature. Suicide Life-Threat. 2008;38:576-91.

[3] Kim DW, Jeong KY, Kim KS. Psychological scales as predictors of emergency department hospitalizations in suicide attempters. Am J Emerg Med. 2018;36:93-9.

[4] Salles J, Calonge J, Franchitto N, Bougon E, Schmitt L. Factors associated with hospitalization after self-poisoning in France: special focus on the impact of alcohol use disorder. BMC psychiatry. 2018;18:287.

[5] Courtet P, Jaussent I, Genty C, Dupuy A-M, Guillaume S, Ducasse D, et al. Increased CRP levels may be a trait marker of suicidal attempt. Eur Neuropsychopharm. 2015;25:1824-31.

[6] Suarez E, Sundy J. Novel markers of inflammation and their relevance to depression: the unique relation of the neutrophil: lymphocyte ratio
(NLR) and the cortisol: C-reactive protein (CORT/CRP) ratio to an intermediate phenotype of major depressive disorders (MDD). Brain Behav Immun. 2017;66:e11-e2.

[7] Demir S, Atli A, Bulut M, İbiloğlu AO, Güneş M, Kaya MC, et al. Neutrophil-lymphocyte ratio in patients with major depressive disorder undergoing no pharmacological therapy. Neuropsych Dis Treat. 2015;11:2253.

[8] Meydaneri GG, Meydaneri S. Can Neutrophil Lymphocyte Ratio Predict the Likelihood of Suicide in Patients with Major Depression? Cureus. 2018;10.

[9] Brook M, Hilty DM, Liu W, Hu R, Frye MA. Discharge against medical advice from inpatient psychiatric treatment: a literature review. Psychiat Serv. 2006;57:1192-8.

[10] Alfandre DJ. "I'm going home": discharges against medical advice. Mayo Clin Proc. 2009;84:255-260.

[11] Hwang SW, Li J, Gupta R, Chien V, Martin RE. What happens to patients who leave hospital against medical advice? CMAJ. 2003;168:417-20.

[12] Jeffer E. K. Against medical advice: Part I, a review of the literature. Mil Med. 1993;158:69-73.

[13] Misson H, Mathieu F, Jollant F, Yon L, Guillaume S, Parmentier $\mathrm{C}$, et al. Factor analyses of the Suicidal Intent Scale (SIS) and the Risk-Rescue Rating Scale (RRRS): toward the identification of homogeneous subgroups of suicidal behaviors. J Affect Disorders. 2010;121:80-7.

[14] Shin H, Kim HJ, Kim S, Choi S, Oh H, Lee B. Should let them go? Study on the emergency department discharge of patients who attempted suicide. Psychiat Invest. 2018;15:638.

[15] Lee H, Roh S. The relations of alcohol drinking behavior depressive mood and suicidal ideation among Korean adults. Journal of Korean Alcohol Science. 2011;12:155-68.

[16] Suominen K, Lönnqvist J. Determinants of psychiatric hospitalization after attempted suicide. Gen Hosp Psychiat. 2006;28:424-30.

[17] Kim WH, Choi YG, Kim SJ, Cho SH. A study on the effect of the depressive disorder in suicidal attempt aspect. Journal of The Korean Society of Emergency Medicine. 2010;21:670-7.

[18] Suominen KH, Isometsä ET, Lönnqvist JK. Attempted suicide and psychiatric consultation. Eur Psychiat. 2004;19:140-5.

How to cite this article: Minho Cho, Hyejin Kim. Factors Associated with Psychiatric Ward Admission in the Emergency Department after a Suicide Attempt: the Risk-Rescue Rating Scale (RRRS) and Biochemical Markers. Signa Vitae. 2020;16(1):97104. doi:10.22514/sv.2020.16.0013. 